\title{
Implementation of New Method to Generate a Key in Automatic Variable Key for Perfect Security
}

\author{
Moumita Das ${ }^{1}$, Rajat Subhra Goswami ${ }^{2}$, Chandan Tilak Bhunia ${ }^{3}$ \\ 1, 2, 3 Department of Computer Science \& Engineering, \\ National Institute of Technology, Arunachal Pradesh, 791110, INDIA \\ ${ }^{1}$ moumita.das23@gmail.com, ${ }^{2}$ rajat.nitap@gmail.com, ${ }^{3}$ ctbhunia@vsnl.com
}

\begin{abstract}
Automatic variable key is a technique to generate time variant key. In this paper, we have proposed a new key generation technique. The proposed technique provides higher level of security compared to that provided by other key generating schemes. The newly generated keys in the technique are more random.
\end{abstract}

Keywords: Randomness, Standard Deviation, Alternating and Shifting Automatic Variable Key, Computing and Shifting Automatic Variable Key, Decimal Shifting Automatic Variable Key, Automatic Variable Key

\section{Introduction}

The fundamental research of Shannon [1-2] on perfect security proposed that if the key is made variable from data to data or session to session in data communication, perfect security can be realized. The first practical approach of perfect security is due to Automatic Variable Key (AVK), proposed by Bhunia [4-7] in the year 2006. There are many researchers like Goswami, Banerjee and Dutta [8-9] who proposed more secure techniques based on Automatic Variable Key (AVK). Basic fundamentals of AVK technique is based on the following procedure:

Let $K_{0}$ be the initial key that will be exchanged between sender and receiver in a secret mode like RSA. Subsequent keys for different data $D_{i-1}$ will be automatically generated between sender and receiver as follows:

$K_{i}=K_{i-1} \oplus D_{i-1} \forall \mathrm{i}>0$.

Where $K_{i-1}$ is the previous key and $K_{i}$ is the generated key.

The key is made variable with exchanged data between sender and receiver. The generated new key is used for exchanging next data.

In Computing and Shifting Automatic Variable Key [14] technique proposed by Goswami in the year 2013, the key is made variable by agreement that creates new key for each data.

Subsequent keys for different data $\left(D_{i-1}\right)$ will be generated as per the following equation

$K_{i}=$

$\bigoplus D_{i-1}^{i} \forall i>0$

Where $K_{i-1}^{\sigma}=$ bit wise right shifted key $K_{i-1} /$ number of shift will be the number of 1's present in $K_{i-1}$ and $D_{i-1}^{r}=$ bit wise left shifted data $D_{i-1} /$ number of shift will be the number of 1 's present in $D_{i-1}$.

In Decimal Shifting Automatic Variable Key [11] technique the key is made by another agreement that creates another new key.

$K_{i}=K_{i-1}^{i} \oplus D_{i-1} \quad \forall i>0$. 
Where $K_{i-1}^{r}=$ bit wise right shifted key $K_{i-1} /$ the number of shift will be corresponding decimal value of $K_{i-1} \oplus D_{i-1}$.

The newly generated key is used subsequently for exchange of data.

In Alternating and Shifting Automatic Variable Key [16] technique the key is made variable by an agreement that also creates new key for each data.

Initial key $K_{0}$ is exchanged between sender and receiver and subsequent key $K_{i}\left(i^{\text {th }}\right.$ stage) is generated by both sender and receiver as follows:

$K_{i}=K_{i-1}^{v} \oplus D_{i-1} \quad \forall \quad \mathrm{i}>0$

$K_{i+1}=K_{i} \oplus D_{i-1}^{v} \quad \forall \mathrm{i}>0$

Where $K_{i-1}^{v}=$ block wise shift $K_{i-1} /$ the number of shift will be total length of $K_{i-1}$ divided by 2 .

and $D_{i-1}^{*}=$ block wise shift $D_{i-1} /$ the number of shift will be total length of $D_{i-1}$ divided by 2 .

\section{Proposed Scheme}

We have proposed a new technique to generate new key in Automatic Variable Key. The main purpose is to generate new key to enhance more security by making more randomness between two successively generated keys.

The proposed technique is as follows:

Step 1: $K_{0}=$ initial key and $D_{0}=$ first data

Step 2: Count Number of 1's and 0's in the previous key $\left(K_{i-1}\right)$

Step 3: Count Number of 1's and 0's in the previous data $\left(D_{i-1}\right)$

Step 4: $K_{1}^{*} \leftarrow K_{0} \oplus D_{0}$.

Step 5: $K_{1} \leftarrow \operatorname{CLS}\left(K_{1}^{*}\right)$

Step 6: $K_{2} \leftarrow K_{1} \oplus D_{1}$

Step 7: $K_{2} \leftarrow C L S\left(K_{2}\right)$

Continue this procedure until dataset $\neq \emptyset$

Step 8. Exit

Where CLS $\left(K_{i}^{\prime}\right)$ is circular left shift of $K_{0}^{\prime}$ by n bits.

The above mentioned technique may be named as LSAVK (Left Shifting Automatic Variable Key)

\section{Example:}

Let us assume, Initial key $\left(\begin{array}{c}K_{0} \\ 0\end{array}=\right.$ $0110011100111101111000101001000011110001001000 \quad 001010011010001100$ and initial data $\left(D_{0}\right)=0100011001101111011100100111011101$ 100001011100100110010000100000

To generate key $K_{1}^{*}$, initial key $\left(K_{0}\right)$ perform the XOR operation with data $D_{0}$ $K_{1}^{*} \leftarrow K_{0} \oplus D_{0}$

$0110011100111101111000101001000011110001001000001010011010001100 \oplus 010001$

$1001101111011100100111011101100001011100100110010000100000=0010000101010$ 010100100001110011110010000010100101100001010101100

$K_{1} \leftarrow$ CLS $\left(K_{1}^{*}\right)$ [1 bit circular left shift of $\left.K_{1}^{\prime}\right]$

$K_{1}=0100001010100101001000011100111100100000101001011000010101011000$

c) $K_{2} \leftarrow K_{1} \oplus D_{1}$ 0100001010100101001000011100111100100000101001011000010101011000

$\oplus 0110010101110010011100100110111101110010001000000110001101101111=0010$

011111010111010100111010000001010010100001011110011000110111 
$K_{2} \leftarrow \operatorname{CLS}\left(K_{2}^{v}\right)$ [2 bit circular left shift of $K_{2}^{\prime}$ ]

1001111101011101010011101000000101001010000101111001100011011100

and procedure is continued until dataset $\neq \emptyset$

3. Review of Existing Computing and Shifting Automatic Variable Key, Alternating and Shifting Automatic Variable Key, Decimal Shifting Automatic Variable Key, Automatic Variable Key

\subsection{Explanation of Computing and Shifting Automatic Variable Key (CSAVK)}

Let us assume that sender sends initial data $D_{0}=01011011010111100111000101011$ 00101011000010101011111011001110011 in encrypted through initial key $K_{0}=1110110001010111001011101101110011011110111110001001101011000110$. As per Computing and Shifting Automatic Variable Key technique in equation number (2) the new key will be generated as:

$K_{1}=1110001001011100000010101101000011010011111110000011100111100000$ and data will be generated as:

$D_{1}=1001010110000101010111110110011100110101101101011110011100010101$

Data has been changed by left shifting the previous data $\left(D_{0}\right)$ up to the total no of 1 's present and key has been changed by right shifting the previous key $\left(K_{0}\right)$ up to the total no of 1's present in key.

$K_{1}^{\sigma} \leftarrow K_{1} \oplus D_{1}$

$1110001001011100000010101101000011010011111110000011100111100000 \oplus 1001$ 010110000101010111110110011100110101101101011110011100010101

$K_{1}^{\sigma}=0010101110100011111011101101110000100000011111100101000000100010$

\subsection{Explanation of Decimal Shifting Automatic Variable Key (DSAVK)}

Let us assume that sender sends initial data $D_{0}=0000000000000000000000000000$ 000000000000000000000000000000000000 in encrypted through initial key $K_{0}=0000011000000110000001100000011000000110000001100000011000000110$. By the Decimal Shifting Automatic Variable Key technique in equation number (3) the new key will be generated by right shift operation and key will be as: $K_{1}=000001100000011000000110000001100000011000000110000001100000011000$

$K_{1} \leftarrow K_{0} \oplus D_{0}$

$0000011000000110000001100000011000000110000001100000011000000110 \quad \oplus$ 0000000000000000000000000000000000000000000000000000000000000000

$K_{1}=0000011000000110000001100000011000000110000001100000011000000110$

\subsection{Explanation of Alternating and Shifting Automatic Variable Key (ASAVK)}

Let us assume that sender sends initial data $D_{0}=0000000000000000000000000$ 000001101101011111111000000000000001010 in different form key $K_{0}=01111100$ 100100110011110111011111111110111101101 01010111110001000. By the Alternating and Shifting Automatic Variable Key technique in equation number (4-5) the next key will be generated as: 1001000000100110101011111000000100010111011 011110011110111010110

\subsection{Explanation of Automatic Variable Key (AVK)}

Let us assume that sender sends initial data $D_{0}=10111100101001001$ 11101100110001100111010110111101001111111111011 in different form 
key $K_{0}=11111000000110110111000111110000110110100100100100011000111011000$ 01100100. By the Automatic Variable Key technique in equation number (1) the new key will be generated as: 010001001011111110000111100100111110000010010111100001 1100010111

\section{Performance Analysis}

For performance analysis of proposed (Left Shifting Automatic Variable Key) we have taken initial key as $K_{0}=01100111001111011110001010010000111100010010000010$ 10011010001100 with following three datasets:

Dataset $1="$ Forward error correction is the process in which the receiver tries to get the message by using redundant bits where as in retransmission receiver asks the sender to resend the message".

Dataset 2 ="Objective of security under a key would be achieved when any one authorized to exchange data gets a key".

Dataset 3="Frequency analysis is based on the fact that, in any given stretch of written language, certain letters and combinations of letters occur with varying frequencies".

Using the same initial key and data set pairs we have found the randomness and standard deviation curves portrayed from Figure 1-6. For analysis, we have assumed randomness is a parameter which is a measure of amount of variation made between successive keys. For example if $K_{i}=10101011$ and $K_{i+1}=00111010$. So the randomness is 3.These results are found more productive and superior among other techniques as per literature survey.

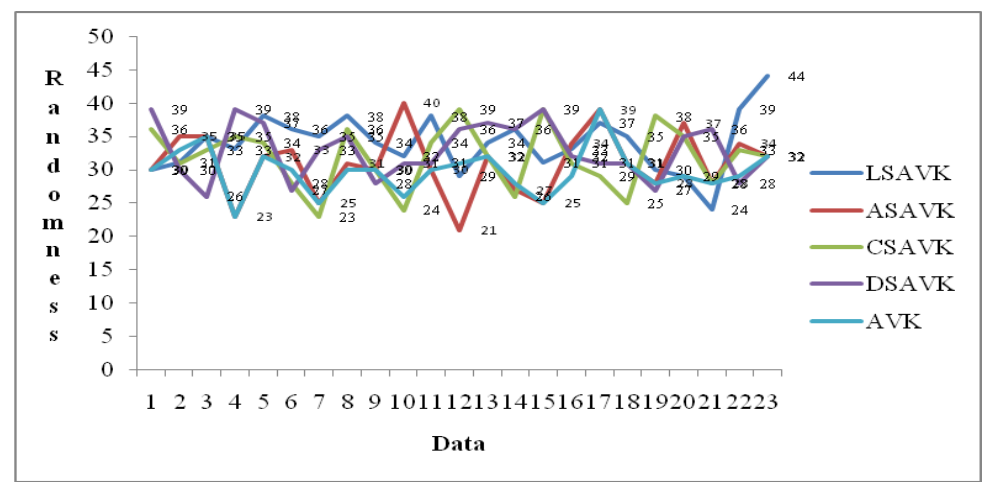

Figure 1. Randomness of Successive Keys of Different Techniques for Dataset1

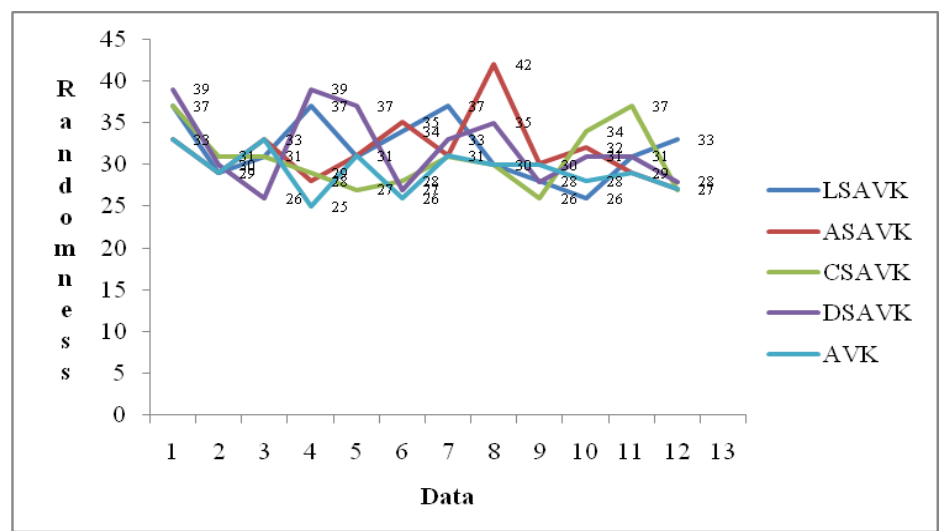

Figure 2. Randomness of Successive Keys of Different Techniques for Dataset2 


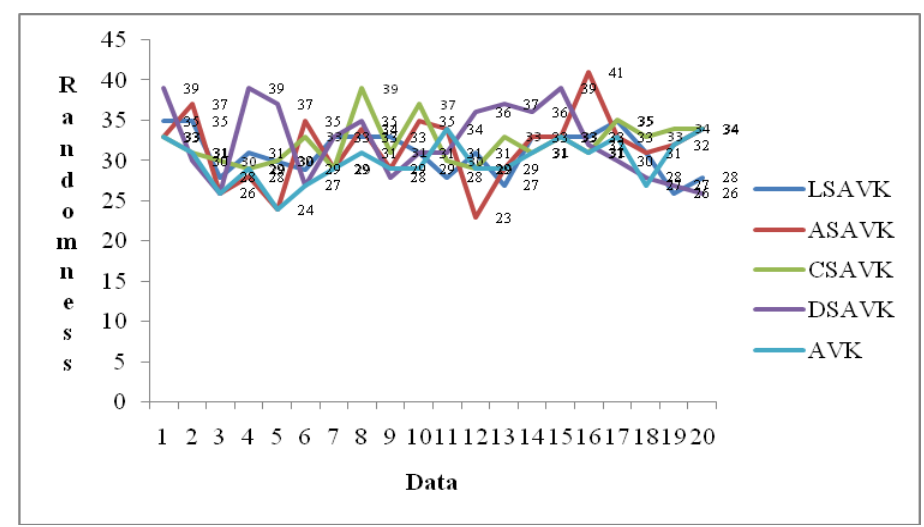

Figure 3. Randomness of Successive Keys of Different Techniques for Dataset3

\section{Comparison}

We have proposed a technique call Left Shifting Automatic Variable Key and we compared the average randomness and standard deviation of Left Shifting Automatic Variable Key technique with other key generation techniques invented by various other researchers such as Alternating and Shifting Automatic Variable Key, Computing and Shifting Automatic Variable Key, Decimal Shifting Automatic Variable Key, and Automatic Variable Key. The randomness and standard deviation were calculated by running programs and results so obtained are portrayed in figures.

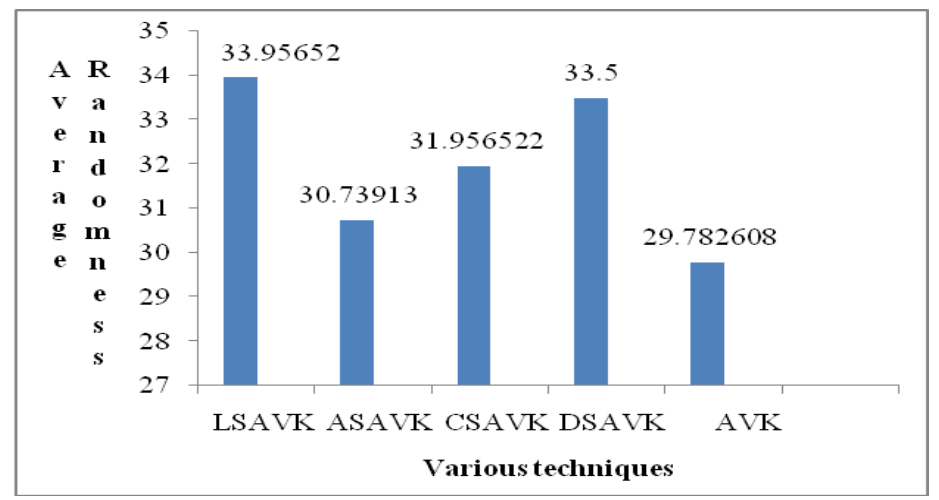

Figure 4. Comparison of Average Randomness for Different Techniques for Dataset1 


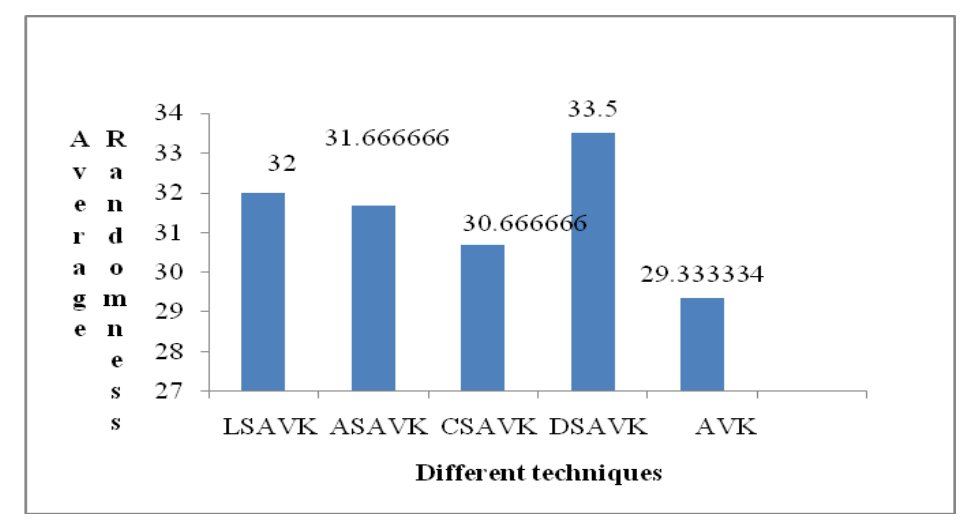

Figure 5. Comparison of Average Randomness for Different Techniques for Dataset 2

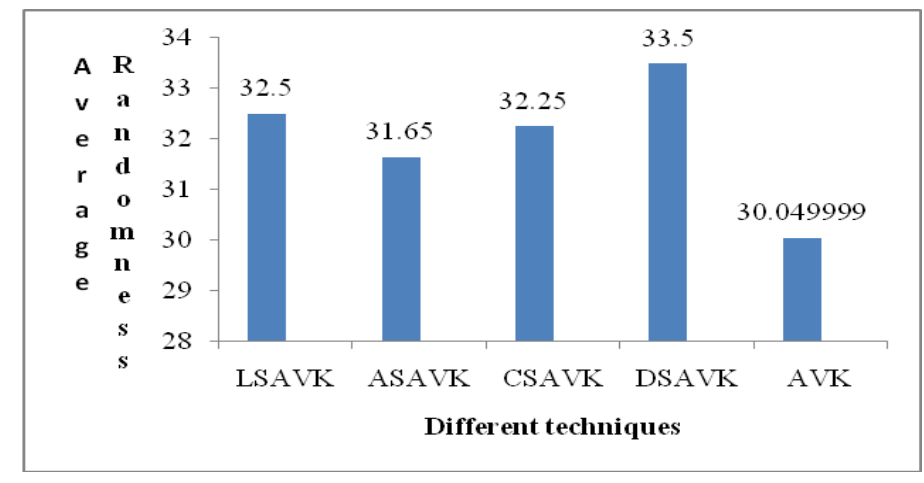

Figure 6. Comparison of Average Randomness for Different Techniques for Dataset 3

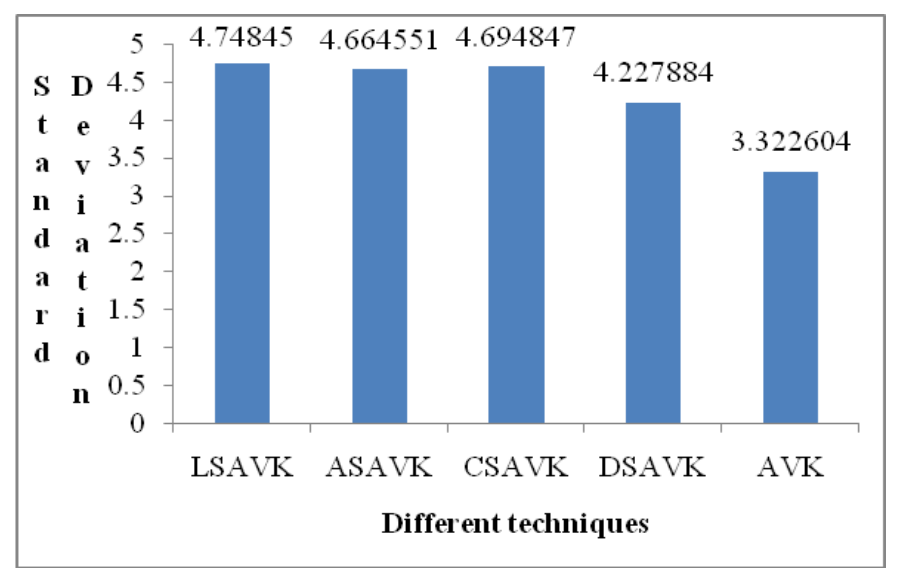

Figure 7. Comparison of Standard Deviation for Different Techniques for Dataset 1 


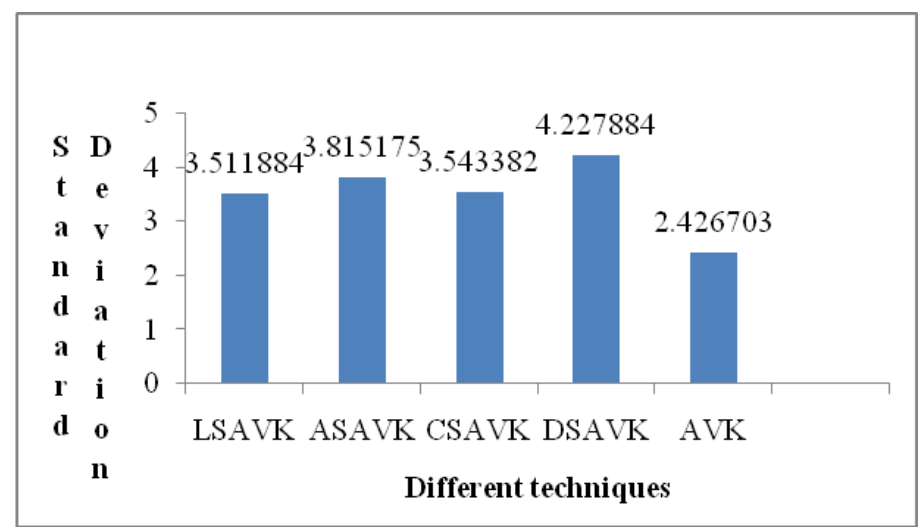

Figure 8. Comparison of Standard Deviation for Different Techniques for Dataset 2

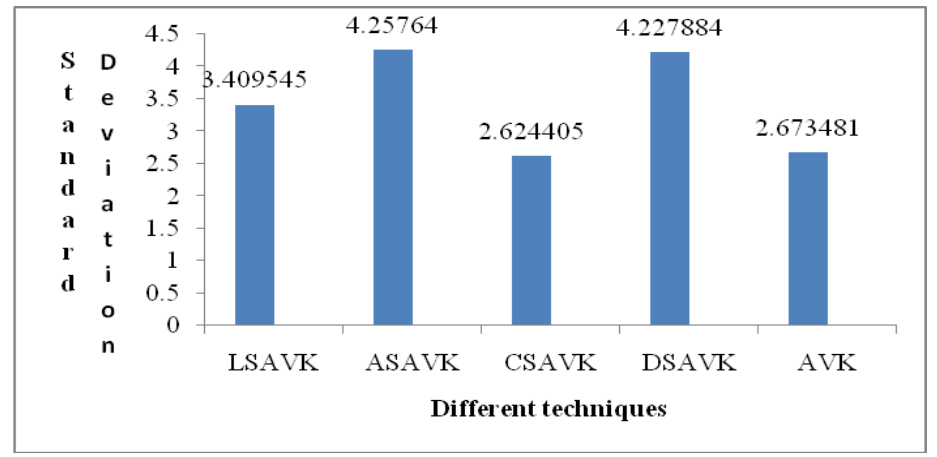

Figure 9. Comparison of standard Deviation for Different Techniques for Dataset 3

To find the superiority of the proposed technique compared to various other techniques of Automatic variable Key such as Alternating and Shifting Automatic Variable Key, Computing and Shifting Automatic Variable Key, Decimal Shifting Automatic Variable Key and Left Shifting Automatic Variable Key, we have computed the average randomness and standard deviation of generated keys.

The comparison of average randomness and standard deviation portrayed in Figure 4-9.

From the graph of average randomness \& standard deviation the following observations may be inferred:

- For similar dataset (dataset1, dataset2 and dataset3) and initial key, average randomness is varying in different session of secure data transmission for different techniques of Automatic Variable key.

- In some cases, it is found that the superiority of Left Shifting Automatic variable Key technique is higher than other techniques. In case of dataset1 this technique is higher than other techniques but in case of dataset 2 and dataset 3 it is vice versa.

- Standard deviation of Left Shifting Automatic Variable Key for dataset1 is higher than other techniques. In case of dataset 2 and dataset 3 the standard deviation of other techniques are higher than the proposed technique. 


\section{Conclusion}

The generated key of Left Shifting Automatic Variable Key, Computing \& Shifting Automatic Variable Key, Alternating \& Shifting Automatic Variable Key, Automatic Variable Key and Decimal Shifting Automatic Variable Key are well established. After analyzing the experiments, it is found that the security ensured by Left Shifting Automatic Variable Key for dataset1 is much higher than other techniques. However, in case of dataset2, Decimal Shifting Automatic Variable Key is found to be superior to other techniques and similarly, in case of dataset3, Alternating and Shifting Automatic Variable Key technique is superior to. Therefore, from the experiments we can infer that the average randomness depends upon the dataset which we are used during data transmission. The superiority of Left Shifting Automatic Variable Key implies that the new technique can be applied with standard algorithms like RSA, AES and DES for encryption and decryption there by increasing the level of security to the next level in real applications.

\section{References}

[1] C E Shannon, "Communication Theory of Secrecy System", the Bell System Tech J, 1949.

[2] C E Shannon, "A mathematical Theory of Communication", Bell System Tech J, 27, 379-423,623-656, 1948.

[3] M. Diffie, E. Hellman, "Exhaustive Cryptanalysis of the no of bits Data Encryption Standard", Computer, pp.74-84, 1977.

[4] C. T. Bhunia, "New approach for selective AES towards tackling error propagation effect of AE", ASIAN Journal of Information Technology, volume 5990, pp.1017-1022, 2006

[5] P.Chakraborty, C.T.Bhunia, "A novel approach towards realizing optimum data transfer and AVK in Cryptography", International journal Computer Science \& Network security, Korea, volume 8, No 5, pp.241-250, 2008.

[6] C.T.Bhunia, "Information Technology, Network and Internet", New age publication.

[7] C.T.Bhunia, "Implementation of AVK with chaos theory and studied thereof", J IUP Computer Science volume V, No 4, pp.22-32, 2011.

[8] B. K. Singh, S.Banerjee, M.P.Dutta, C.T.Bhunia, "Generation of Automatic Variable key to make secure communication", International conference on recent cognizance in wireless communication\& image processing-ICRCWIP, 2014.

[9] M.P.Dutta, S.Banerjee, C.T.Bhunia, "Two new schemes to generate automatic variable key to achieve the perfect security in insecure communication channel", In preceding of the International Conference on Advanced Research in Computer Science Engineering \&Technology (ICARCSET, Eluru, India), 2015.

[10] R Subhra Goswami, S Chakraborty, C. T. Bhunia "Various new methods of Implementing Automatic Variable Key", Preceding of the 2nd international Conference Advanced Computer science and Engineering, pp.149-152, 2013.

[11] R Subhra Goswami, S Chakraborty, C. T. Bhunia, Abhinandan Bhunia, "New approach towards generation of Automatic Variable Key to achieve perfect security", ITNG, IEEE Computer Society, CPS,USA, Las Vegas, pp.489-491, 2013.

[12] R Subhra Goswami, S Chakraborty, C Tilak Bhunia, "Approach towards Optimum data transfer with various Automatic variable Key techniques to achieve perfect security with analysis and comparison", International Journal of Computer Application, Volume 82, Issue 1, 2013.

[13] R Subhra Goswami, S Chakraborty, C. T. Bhunia, Abhinandan Bhunia, "Generation of Automatic variable key under various approaches in cryptography system", J. Institute Engineering, India Ser. B, 94, 4,215-220.

[14] C.T.Bhunia, Rajat Subhra Goswami, Swarnendu Kumar Chakraborty, "A new technique of AVK in achieving perfect security", 100th Indian Science Congress Association 3rd-7th, 2013.

[15] C.T.Bhunia, G. Mondal, S. Samaddar, "Theory and application of time variant key in RSA and that with selective encryption in AES", in preceding of EAIT (Elsevier Publications, Calcutta CSI), pp.219-221.

[16] R Subhra Goswami, S Chakraborty, A Bhunia, C. T. Bhunia, "New techniques for generating of Automatic variable Key in achieving perfect security", J. Institute Engineering, India Ser. B, 95, 3, pp. 197-201, 2014.

[17] Rajat Subhra Goswami, S. Banerjee, M.P.Dutta, C.T.Bhunia, "Absolute key variation technique of AVK in Cryptography", In preceding SIN'15 of the 8th International Conference on security of Information and Networks(Sochi, Russia),8th-10th September, 2015. 
[18] Rajat Subhra Goswami, Swarnendu Chakraborty, Chandan Tilak Bhunia, "A study to examine the Superiority of CSAVK, AVK over conventional encryption with a single key", International Journal of Security and Its Applications, Volume 10, No 2, pp.279-286, 2016.
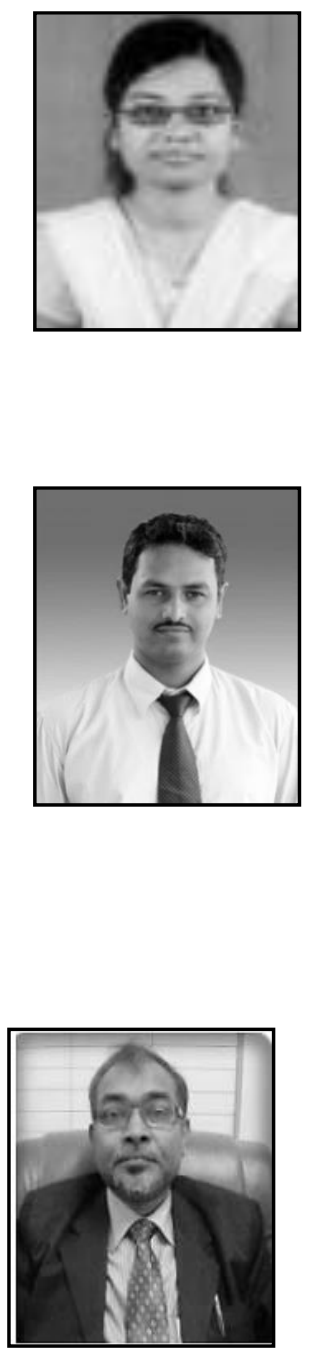

\section{Authors}

Moumita Das, earned her M. Tech degree in Information Technology from Bengal Engineering \& Science University, Shibpur in 2013. She has teaching experience more than four years. She worked as a junior research fellow in the department of Electronics \& Communication Engineering at National Institute of Technology, Arunachal Pradesh, Govt. of India. She is currently pursuing $\mathrm{PhD}$ in the department of Computer science \& Engineering and working as a Guest Lecturer in the department of Computer science \& Engineering at National Institute of Technology, Arunachal Pradesh, Govt. of India.

Dr. Rajat Subhra Goswami, working as an Assistant Professor in the department of Computer science \& Engineering in National Institute of Technology, Arunachal Pradesh, Govt of India. He is having more than 8 years of teaching experience. His research areas are cryptography and information security. He is the author of 20 peer reviewed publications. In 2013, he got international travel support grand from department of Science \& Technology to present one of his research papers at Los Angeles, USA. In 2013, he was awarded as best teacher for the academic year 2012-2013. He received his $\mathrm{PhD}$ degree from National Institute of Technology, Arunachal Pradesh in 2015.

Professor Chandan Tilak Bhunia earned his B. Tech in Radio Physics and Electronics in 1983 from Calcutta University then joined DVC of Govt. of India as telecommunication engineer. He got M. Tech degree in Radio Physics and Electronics in 1985 and then joined North Bengal University as a lecturer of computer science \& application in 1988, and became Assistant Professor of electronics \& communication engineering at the North Eastern Regional Institute of Science \& Technology (NERIST) of Govt. of India in 1990. He got his $\mathrm{PhD}$ in computer science \&engineering from the Jadavpur University. He became full Professor in 1997 at NERIST where he was HOD of ECE \& CSE for about 6yrs and Dean (Academics/Post Graduate Studies) for about 1.5 years. He then switched to private engineering colleges from 1999 to 2003 as HOD, Deputy Director and Director. Lastly, he was a full Professor of computer science \& engineering of the Indian School of Mines (Deemed University) of Govt of India. He was a senior Professor and Dy Director (Acad) of Haldia Institute of Technology. He has extensively visited foreign countries, namely China, Italy, Singapore, UK and Bangladesh on several assignments including BOYSCAST Fellowship and ICTP senior associate ship. He has published around 200 research papers and technical articles/reports in national/international journals/magazines/seminars. He is the author of the books a) "Introduction to Knowledge Management" published by Everest Publishing House, Pune in 2003 and b) "Information Technology, Network and Internet", published by the New Age International 
International Journal of Security and Its Applications

Vol. 10, No. 4 (2016)

Publishers, New Delhi. He is fellow of IETE and the IE (I) and a senior member of IEEE and CSI. He is currently as a Director of National Institute of Technology, Arunachal Pradesh. 